\title{
Case Report 2 :Rabies Through Organ Transplant
}

\author{
Mariam Al Fadhli ${ }^{1}$, Saroj Bella Grover ${ }^{1}$, Mohamed Ahmed Saraya ${ }^{2}$ \\ ${ }^{1}$ Department of the Medicine, Infectious Diseases Hospital, Kuwait. \\ ${ }^{2}$ Department of Tropical Medicine, Faculty of Medicine, Zagazig University, Zagazig, Egypt.
}

Corresponding Author Mariam Al-Fadhli

Mobile:

+96599660936

E mail:

medicine209@gmail.c om

Key words: Rabies, Transmission, recipient, infectious

\section{ABSTRACT}

The rabies virus causes fatal encephalitis and can be transmitted through tissue or organ transplantation. In March 2015, a kidney recipient with no reported exposures to potentially rabid animals died from rabies after transplantation.

\section{INTRODUCTION}

Rabies is an endemic fatal Zoonotic disease commonly transmitted to humans through contact (bites and scratches) with infected animals. Human rabies is rare in Kuwait. Most cases of rabies that have occurred in Kuwait are imported cases, having history of exposure to a rabid animal in their country. Rabies transmission via solid organ transplantation though reported in different parts of the world is rare. We report a case of rabies in a kidney transplant recipient with no reported exposure to a potentially rabid animal. This patient developed fatal encephalitis and died. This is the first case of rabies in an organ recipient in Kuwait.

\section{CASE DESCRIPTION}

A 5 year old Kuwaiti child presented with history of high grade fever and status epileptics. She was a known case of renal failure, since birth. 3 months prior to admission the child had been transplanted kidney from an Indian male who had died due to encephalitis, the cause for which was not established.

Anticonvulsants drugs were started, in addition to cefotaxime and acyclovir after doing CT scan brain, and lumbar puncture which were normal. She was put on mechanical ventilation, as had generalized convulsions and tremors. She was febrile, had tachycardia, was well hydrated, BP 120/70 mmHg. The examination of the heart, chest and abdomen were unremarkable. Complete blood counts, renal function tests, liver function tests, biochemistry, blood culture, were normal. PCR for CMV was high but other virology study such as HHV6, HHV7, HHV8, EBV, Enterovirus, Coxackie were IgM negative. She later received injection meropenum, gangcylovir, intravenous immunoglobulin and injection ambisone. Later the child died due to respiratory distress.

\section{DISCUSSION}

Our case has received organ (kidney) from a donor who had recently died of encephalititis, the cause for which had not been established. Also from the same donor, recipients who has received kidney, heart, liver, developed signs and symptoms similar to the one that the donor had presented with and died. Suspicion of Rabies was raised and the source was suspected to be the donor.

Rabies is not endemic in Kuwait. Most cases of Rabies are imported cases with the history of the patient being exposed to a rabid animal in their own country. Within the last 10 years we had only 3 cases, with the last one, being 2 years back. 
Hence, in our case though the patient had presented with features of encephalitis, diagnosis of Rabies was suspected retrospectively when cluster of cases with similar signs and symptoms occurred raising the suspicion of Rabies in the other organ recipients. An investigation was initiated. The father of the donor was contacted in India and he indicated that the donor was bitten by a street dog in his home town, prior to his return to Kuwait and the dog had died. Furthermore the biopsy for rabies taken from the recipient of the organ had confirmed the diagnosis. The two corneal recipients from the same donor were not infected after their grafts were removed and were given rabies post exposure prophylaxis, and they are being followed closely.

Rabies is a preventable viral disease of mammals, commonly transmitted to humans through contact (bites and scratches) with infected animals. One important mode of no bite transmission is person to person via organ transplantation particularly in corneal transplantation [1-7] such transmission has occurred in eight recipients in five countries like Thailand (two cases), India (two cases), Iran (two cases) U.S.A (one case) and France (one case) [8].

Human rabies due to organ transplantation has been reported, though quite rare. In 2004, CDC confirmed the first reported cases [9]. Although rabies transmission had occurred previously through cornea transplants, this was the first report of rabies transmission via solid organ transplantation. The organ donor had undergone routine eligibility screening, including laboratory testing. One of the organ recipient died during transplant surgery and the other three recipients died later of rabies. It was discovered later (after the death of the donor) from the friends of the donor that the later had recently been bitten by a bat. A similar case had happened in Maryland. The man died from Rabies after receiving a kidney from an infected donor [9]. The recipient had no reported animal exposure. CDC laboratories tested tissue samples from the donor and the recipient who had died to confirm diagnosis of rabies through organ transplantation. Both had the same type of virus - a raccoon type [9].

A similar rabies transmission through organ transplantation occurred in Germany [9]. Six recipients received organs or tissues from a donor with rabies. Two recipients receiving donor corneas were not infected after their grafts were removed. Recipients who received lung, kidney and combined Kidneys and pancreas died. The liver recipient had been previously vaccinated against rabies and survived.

Usually if Rabies is not clinically suspected laboratory testing for Rabies is not routinely performed, as it is difficult for doctors to confirm results in the short time, the organs have to be kept viable for the recipients. This happened in our case.

Given the mortality with transplant associated encephalitis, it becomes imperative to suspect rabies as a cause for infectious encephalitis among organ donors, especially if the donor is from endemic area.

\section{CONCLUSION}

Rabies can be difficult to diagnose especially in countries where it is a rare disease. There is a lack of awareness of rabies being a cause of obscure behavioral and neurologic manifestation especially if a history of bite does not exist. In that event the disease may easily be mistaken for other neurological or psychiatric condition.

We suggest that any organ procurement organization should add a question for exposure to any animal in the screening questioner, even though it may be a rare disease in their countries. What also needs to be looked into more closely that organs from donors especially those from endemic areas of Rabies presenting with infectious encephalitis should only be used in extreme circumstances, especially when screening for rabies prior to donation cannot be done due to lack of facilities for screening.

\section{REFERENCES}

1. Chang HG, Eidson M, Noonan - Toly C, et al. Public Health impact of reemergence of rabies, New York. Emerg Infect Dis 2002; 8:909-13.

2. Rupprecht CE, Briggs D, Brown C, et al. Evidence for a 4 - dose vaccine schedule for human rabies post exposure prophylaxis in previously non-vaccinated individuals. Vaccine 2009; 27:7141-8

3. Dutta JK. Rabies transmission by oral and other no -bite routes. J Indian Med Assoc. 1998; 96:359

4. Schoenstadt A. Health information brought to life, rabies transmission. Available at: http://rabies. emedtv.com/rabies/rabies - transmission. html. 
5. CDC - Human rabies preventation - United States 2008. Recommendations of the Advisory Committee on Immunization Practices (ACIP). MMWR Recomm Rep 2008; 57 (RR-3): 1-28

6. Javadi MA, Fayaz A, Mirdehghan SA, Ainollahi B. Transmission of Rabies by Corneal graft. Cornea 1996; 15(4) 431-3.

7. Johnson N, Lipscombe DW, Stott R, et al. Investigation of a human case of Rabies in the United Kingdom. J Clin Virol 2002; 25: 351-6
8. Susan S, Ahmad F, Pooneh R, at el. Six fatal cases of classical rabies virus without biting incidents, Iran 1990 - 2010. J Clin Virol 2012; 54:251-254

9. CDC - News (March 15, 2013): Q\&A - Human Rabies due to organ transplantation 2013 http://www.cdc.gov/rabies/resources/news/201303-15.html. 